\title{
Persistence of Fear Memory Depends on a Delayed Elevation of BAF53b and FGF1 Expression in the Lateral Amygdala
}

\author{
${ }^{\circledR}$ Miran Yoo, ${ }^{1,2}{ }^{\circledR}$ Seongwan Park, ${ }^{1}{ }^{\circ}$ Inkyung Jung, ${ }^{1}$ and ${ }^{\circledR}$ Jin-Hee Han ${ }^{1,2}$ \\ ${ }^{1}$ Department of Biological Sciences, KAIST (Korea Advanced Institute of Science and Technology), Daejeon 34141, Republic of Korea, and ${ }^{2}$ KAIST \\ Institute for the BioCentury, KAIST (Korea Advanced Institute of Science and Technology), Daejeon 34141, Republic of Korea
}

Endurance represents a highly adaptive function of fear memory and a major cause of maladaptive fear- and anxiety-related mental disorders. However, less is known about the mechanisms underlying the persistence of fear memory. The epigenetic gene regulation recently emerged as an important mechanism for memory persistence. In the previous study, we found that BAF53b, a neuron-specific subunit of BAF chromatin remodeling complex, is induced after auditory cued fear conditioning in the lateral amygdala (LA) and is crucial for recent fear memory formation. In this study using mice of both sexes, we report a delayed induction of BAF53b in the LA $48 \mathrm{~h}$ after auditory fear conditioning and its critical role for the persistence of established fear memory. To specifically block the delayed but not the early induced BAF53b function, we used a postlearning knock-down method based on RNAi. The transient knockdown of Baf53b using siRNA in the lateral amygdala $24 \mathrm{~h}$ after cued fear conditioning led to specific impairment of remote but not recent memory retrieval. RNA-sequencing analyses identified fibroblast growth factor 1 (FGF1) as a candidate downstream effector. Consistently, postlearning administration of FGF1 peptide rescued memory persistence in Baf53b knock-down mice. These results demonstrate the crucial role of BAF53b and FGF1 in persistent retention of fear memory, giving insights into how fear memory persistently stored through consolidation processes and suggest candidate target for treating mental disorders related to traumatic memory.

Key words: BAF53b; conditioning; fear; FGF1; memory; persistence

Significance Statement

It is still unclear how once consolidated memory persists over time. In this study, we report the delayed induction of nucleosome remodeling factor BAF53b in the lateral nucleus of amygdala after fear learning and its crucial role for persistence of established memory beyond $24 \mathrm{~h}$ after learning. Our data link the regulation of BAF53b and fibroblast growth factor 1 expression in the amygdala to fear memory persistence. Results from this study open a new direction to understand the time-dependent continuous consolidation processes potentially by a nucleosome-remodeling mechanism enabling long-lasting memory formation and give insights into how to treat mental disorders caused by enduring traumatic memory.

\section{Introduction}

Persistent retention of memory is thought to rely on the stabilization and persistence of synaptic changes via continued consolidation processes over time (McGaugh, 2000; Katche et al., 2013; Dudai et al., 2015; Merlo et al., 2015; Albo and Gräff, 2018). In the process of newly acquired memory being transformed into long-lasting memory, de novo gene expression is required. However, it is not clear how our memory can have such

\footnotetext{
Received Mar. 23, 2020; revised June 24, 2020; accepted July 23, 2020

Author contributions: M.Y., I.J., and J.-H.H. designed research; M.Y. and S.P. performed research; M.Y., S.P., I.J., and J.-H.H. analyzed data; M.Y., S.P., I.J., and J.-H.H. wrote the paper.

This work was supported by the National Research Foundation of Korea (NRF) funded by the government of the Republic of Korea (MSIT; Grant 2018R1A2B3004486). We thank all laboratory members for helpful discussions and comments.

The authors declare no competing financial interests.

Correspondence should be addressed to Jin-Hee Han at han.jinhee@kaist.ac.kr.

https://doi.org/10.1523/JNEUROSCl.0679-20.2020

Copyright $\odot 2020$ the authors
}

persistence despite the short protein turnover rate. It implies the need for a self-perpetuating molecular mechanism for the persistent retention of memory, and the epigenetic mechanism has emerged as an important one. The epigenetic mechanisms of DNA methylation, and post-translational histone modifications and exchange have been implicated in consolidation and persistent storage of memory (Day and Sweatt, 2011; Campbell and Wood, 2019). However, recent works have just begun to show the possible involvement of ATP-dependent chromatin remodeling in long-term memory formation (Vogel-Ciernia et al., 2013; White et al., 2016; Yang et al., 2016; Yoo et al., 2017; Schoberleitner et al., 2019). BAF53b, a postmitotic neuron-specific subunit of BAF chromatin remodeling complex, is shown to be critical for the early consolidation process responsible for $24 \mathrm{~h}$ lasting memory (Vogel-Ciernia et al., 2013; White et al., 2016; Yoo et al., 2017). In our prior study, we found that the mRNA level of Baf53b is induced early ( $3 \mathrm{~h}$ ) in the lateral amygdala (LA) and remains increased even $24 \mathrm{~h}$ after cued fear conditioning 

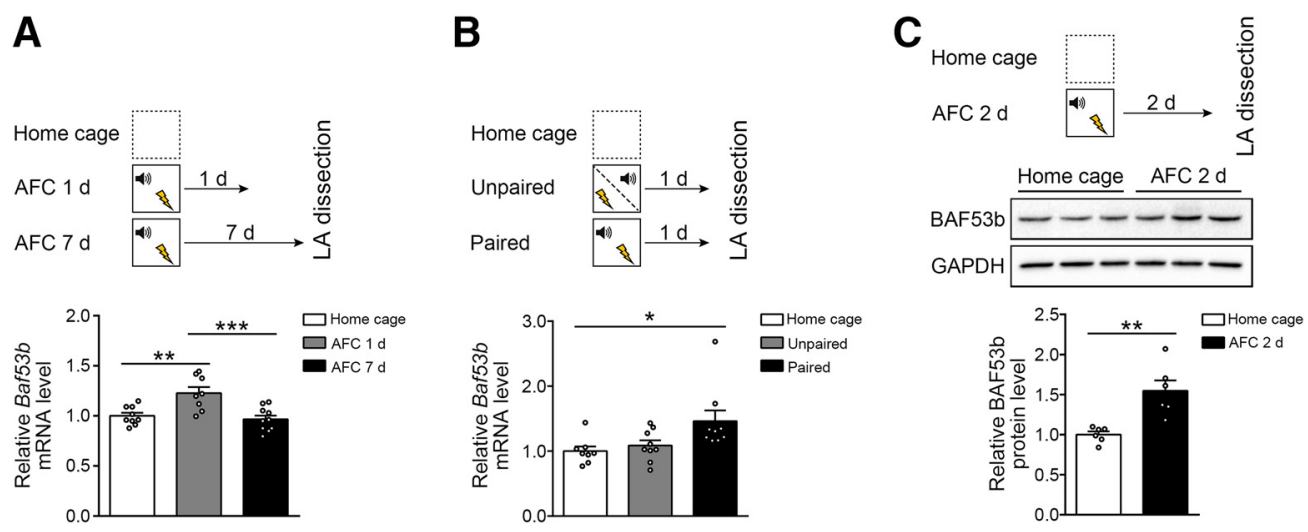

Figure 1. The late post-training induction of BAF53b by auditory cued fear conditioning in the LA. $\boldsymbol{A}, \boldsymbol{B}$, Relative Baf53b mRNA levels in the LA in each experimental condition. The mRNA levels were normalized to the Gapdh mRNA level ( $\boldsymbol{A}$ : home cage, $n=9$; AFC $1 \mathrm{~d}, n=8$; AFC $7 \mathrm{~d}, n=10 ; \boldsymbol{B}$ : home cage, $n=8$; unpaired, $n=9$; paired, $n=9$ ). C, Representative Western blots and quantification. The BAF53b protein levels were normalized to the GAPDH protein level $(n=6 /$ group). Uncropped Western blots are presented in Extended Data Figure 1-1. $n$ values indicate the number of mice. Data are the mean \pm SEM. ${ }^{*} p<0.05,{ }^{* *} p<0.01,{ }^{* * *} p<0.001$.

(Yoo et al., 2017). Previous studies reported similarly delayed induction for several other genes, such as BDNF, and immediate early genes in different learning tasks, and a critical role of such delayed induction for the persistence of memory (Bekinschtein et al., 2007; Katche et al., 2013; Nakayama et al., 2016). Therefore, the late induction of BAF53b in the LA suggests that BAF53b may play a role for memory persistence. However, this possibility has not been tested yet. In this article, we demonstrate the role of BAF53b in remote memory formation by using the RNAi method, which allowed us to block BAF53b induction specifically after recent memory formation. Moreover, using RNA sequencing (RNA-seq) analysis, we identified a putative downstream effector mediating BAF53b function for memory persistence.

\section{Materials and Methods}

Mice. All experiments were performed on adult $129 \times \mathrm{C} 57 \mathrm{BL} / 6$ hybrid mice of both sexes (overall, 189 males, 164 females) that were 23 months old. Mice were group housed (3-5 mice per cage) with free access to food and water at a constant temperature $\left(22 \pm 1^{\circ} \mathrm{C}\right), 40-60 \%$ humidity, and under a $12 \mathrm{~h}$ light/dark cycle. All behavioral experiments were performed during the light phase. Only male mice were used for Figure $1 A$ (and also see Figs. $4 A, B$ and $5 A$ ) because of variability in transcription during the female estrus cycle (DiCarlo et al., 2017). To confirm the results, both sexes were used in Figure $1 B$ (and also see Fig. $4 C$ ). Otherwise, both males and females were used in balance. All experiments were approved by and performed in accordance with the guidelines of the Institutional Animal Care and Use Committee.

Auditory fear conditioning. For training, mice were placed in a conditioning chamber (Coulbourn Instruments) for $2 \mathrm{~min}$, followed by the conditioned stimulus (CS; $2800 \mathrm{~Hz}, 85 \mathrm{~dB}$ pure tone, $30 \mathrm{~s}$ duration) coterminating with the unconditioned stimulus (US; $0.5 \mathrm{~mA}$ foot shock for $2 \mathrm{~s}$ ). They were then allowed to remain in the chamber for an additional $30 \mathrm{~s}$. For the retention sessions, CS-induced freezing was measured in a novel context (Plexiglas plate, a round wall, and a striped wall on the opposite side). The CS was presented to the mice for $3 \mathrm{~min}$ after 2 min of pre-CS. Freezing behavior was automatically scored using FreezeFrame software (Actimetrics). The freezing level during the first 1 min of tone presentation was analyzed to determine the conditioned response to the auditory CS. For contextual fear memory test, mice were placed in the conditioning chamber for $3 \mathrm{~min}$. The freezing level during the $3 \mathrm{~min}$ was analyzed to determine conditioned response to the context. For immediate shock condition, the $2 \mathrm{~s}$ shock was given immediately after mice entering the chamber. For unpaired conditioning, mice were placed in the conditioning chamber for $2 \mathrm{~min}$ followed by the US and subsequently the CS with a $30 \mathrm{~s}$ interval. After the CS, mice remained in the chamber for an additional $30 \mathrm{~s}$.

Quantitative real-time PCR. We anesthetized mice with isoflurane and extracted brains. We sectioned the brains using a vibratome at $300 \mu \mathrm{m}$ thickness in ice-chilled PBS. LA tissues were dissected and stored in Invitrogen RNAlater (catalog AM7020, Thermo Fisher Scientific). Total RNAs were isolated using the mirVANA miRNA Isolation Kit (catalog \#AM1560, Ambion). cDNAs were reverse transcribed using the miScript II RT kit (catalog \#218160, QIAGEN) with the miScript HiFlex buffer. The following primers were used for mRNA quantification: Baf53b: forward, 5'-TCCTGCCTTCTTCTTATGCAA3'; reverse, 5'-CCTGTGGAGCGTCCATTT-3'; Fgfl: forward 5'-CC GAAGGGCTTTTATACGG-3'; reverse, $5^{\prime}$-TCTTGGAGGTGTAAGT GTTATAATGG-3'; Fgf2: forward, 5'-CAACCG GTACCTTGCTAT GA-3'; reverse, $5^{\prime}$-TCCGTGACCGGTAAGTATTG-3'; and Gapdh: for-

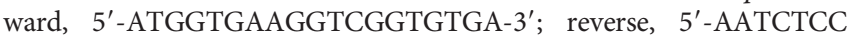
ACTTTGCCACTGC-3'. Quantitative real-time PCR (qRT-PCR) was performed using QuantiTect SYBR Green PCR Kit (catalog \#204143, QIAGEN) with a Rotor-Gene Q (QIAGEN). The expression of each gene was normalized to Gapdh. Relative quantifications between groups were analyzed using the $\Delta \Delta \mathrm{CT}$ method.

Western blot. It was performed as previously described (Yoo et al., 2017). Briefly, we anesthetized mice with isoflurane and extracted brains to prepare $300-\mu \mathrm{m}$-thick coronal brain sections using vibratome. Then we dissected LA tissues and lysed them in $20 \mu \mathrm{l}$ of ice-cold lysis buffer (50 mm HEPES, pH 8.0, $400 \mathrm{~mm} \mathrm{NaCl}, 10 \%$ glycerol, $1 \%$ Triton X-100, $5 \mathrm{~mm}$ DTT) containing a protease inhibitor cocktail (catalog \#11836153001, Roche). The lysates (30-50 $\mu \mathrm{g} / \mathrm{lane})$ were resolved and transferred to PVDF membranes using the Trans-Blot Turbo Blotting System (Bio-Rad). After blocking with $5 \%$ nonfat dried milk in TNTX buffer (50 mu Tris- $\mathrm{HCl}, \mathrm{pH}$ 7.5, $200 \mathrm{~mm} \mathrm{NaCl}, 0.2 \%$ Triton X-100) for $30 \mathrm{~min}$ at room temperature, the membranes were incubated with primary antibodies against BAF53b (1:2000) or BAF45b (1:500) in 3\% bovine serum albumin (BSA) overnight at $4^{\circ} \mathrm{C}$. HRP-conjugated goat anti-mouse IgG light chain or HRP-conjugated goat anti-rabbit IgG were used as secondary antibodies (1:2000). The antibodies were detected using ECL Solution (catalog \#RPN2232, GE Healthcare). The blotted membranes were stripped using Restore Western Blot Stripping Buffer (catalog \#21059, Thermo Fisher Scientific) then stained with antiGAPDH antibody (1:2000) as a loading control. To determine the level of fibroblast growth factor 1 (FGF1) and the phosphorylated form of FGF receptor R1 (pFGFR1) following FGF1 peptide injection, we dissected LA tissue on the ice-cold plate and solubilized hydrophilic proteins and hydrophobic proteins separately by using the Pierce Mem-PER Plus Eukaryotic Membrane Protein Extraction Kit (catalog \#89842, Thermo Fisher Scientific) according to the manufacturer instruction. The hydrophilic lysates were resolved and transferred to PVDF membranes, blotted with anti-FGF1 (1:2000), then stripped, and blotted again 
with anti-GAPDH (1:2000). The hydrophobic membrane proteins were resolved and transferred to PVDF membranes, blotted with antipFGFR1 (1:1000), then stripped, and blotted again with anti-FGFR1 (1:1000). HRP-conjugated goat anti-mouse IgG or HRP-conjugated goat anti-rabbit IgG were used as secondary antibodies (1:2000). The protein levels were analyzed using Image Lab version 6.0.1 and normalized to the GAPDH protein level. The pFGFR1 protein levels were normalized to the total FGFR1 protein levels.

Surgery. Mice were anesthetized with pentobarbital $(83 \mathrm{mg} / \mathrm{kg}$ body weight) by intraperitoneal injection and placed on a stereotaxic frame. Guide cannula (3 mm guide; catalog \#C313GS-5, Plastics One) were chronically implanted into the lateral amygdala bilaterally (anteroposterior $=-1.8 \mathrm{~mm}$, mediolateral $= \pm 3.6 \mathrm{~mm}$, dorsoventral $=-3.0 \mathrm{~mm}$ from the bregma).

siRNA injection. Scrambled, BAF53b, and BAF45b siRNA were purchased from Ambion (catalog \#amb-4390843, \#amb-4390771, assay ID s96445, amb-4390771, and assay ID s15690, respectively). In vivo transfection of siRNA was performed using Invitrogen Invivofectamine 3.0 Reagent (catalog \#INV-IVF3001, Thermo Fisher Scientific). A total of $0.1 \mu \mathrm{g}$ of siRNAs in $0.5 \mu \mathrm{l}$ solution was injected into each side of LA through the internal cannula ( $3 \mathrm{~mm}$ guide with $1 \mathrm{~mm}$ projection; catalog \#C313IS-5, Plastics One) using an infusion pump at a rate of $0.1 \mu \mathrm{l} / \mathrm{min}$ for $5 \mathrm{~min}$ and left for an additional $10 \mathrm{~min}$ for diffusion. During the injection, the mice were allowed to move freely in their home cage.

RNA-seq and data processing. We extracted total RNA using the RNeasy Lipid Tissue Mini Kit (QIAGEN) from the LA tissues of four mice for each group obtained after the behavioral procedures. RNA sequencing libraries were constructed using Illumina Truseq Stranded mRNA LP Kit. Briefly, mRNA was purified by Oligo-dT beads and fragmented through an enzymatic reaction. After fragmentation, cDNA was generated through reverse transcription. The cDNA libraries were constructed and followed by a $75 \mathrm{bp}$ paired-end mode on the Illumina Nextseq 550 platform. External RNA Controls Consortium RNA spikein mixes (catalog \#4456740, Thermo Fisher Scientific) were included for quality assurance. The sequenced reads were aligned to the reference genome $(\mathrm{mm} 10)$ using TopHat 2 with default parameters. The abundance of transcripts was quantified as FPKM (fragments per kilobase per million reads mapped) using Cufflinks based on Gencode M24 gene annotation followed by quantile normalization. We performed two biological replicates of the independent experiments to ensure the result. The reproducibility between biological replicates was measured by hierarchical clustering analysis based on Pearson correlation coefficient of FPKM values between all pairs of samples. We confirmed that all replicates were clustered together with a higher correlation coefficient, ensuring the quality of our RNA-seq result. To investigate dynamic expression in various experimental conditions, we first selected genes showing over twofold changes between the maximum and minimum expression values between samples. Next, we conducted k-means clustering $(\mathrm{k}=3)$ and revealed specifically upregulated genes in naive, Training + scrambled siRNA, and Training + BAF53b siRNA groups, respectivley. Finally, we ranked the upregulated or downregulated genes according to the relative expression between Training + scrambled siRNA and naive siRNA, as illustrated in Table 1. The qRT-PCR validation of the RNA-seq result was performed using independent biological replicates from the RNAseq experiment.

Peptide injection. Recombinant mouse FGF1 (catalog \#4686-FA, R\&D Systems; Uchida et al., 2017) or FGF2 peptide (R\&D Systems, 3139-FB; Graham and Richardson, 2009) was diluted to $10 \mathrm{ng} / \mu \mathrm{l}$ in $0.1 \%$ BSA in $1 \times$ filtered PBS. A total of $0.5 \mu$ l of peptide solution or $0.1 \%$ BSA (vehicle) was injected into each side of LA through internal cannula using an infusion pump at a rate of $0.1 \mu \mathrm{l} / \mathrm{min}$ for $5 \mathrm{~min}$ and was left for an additional $10 \mathrm{~min}$ for diffusion. During the injection, the mice were allowed to move freely in their home cage.

Open field test. Mice were placed in the center of an open field box $(45 \times 45 \times 25 \mathrm{~cm})$. Using a video camera, $30 \mathrm{~min}$ of movement was recorded per mouse, and the total distance that each mouse moved and the frequency with which it crossed the center zone $(22.5 \times 22.5 \mathrm{~cm})$ during 20 min was quantified using EthoVision XT (Noldus).

Elevated plus maze. Mice were placed on the center platform of an elevated plus maze facing one of the closed arms. The maze is made of
Table 1. Top 20 differentially expressed genes whose expressions are increased or decreased in the training + scrambled siRNA group compared with the naive group

Scrambled siRNA/naive BAF53b siRNA/naive

\begin{tabular}{|c|c|c|}
\hline & Scrambled siRNA/naive & BAF53b siRNA/naive \\
\hline \multicolumn{3}{|c|}{ Upregulated gene name } \\
\hline Scgb3a1 & 1.997089204 & 1.47610085 \\
\hline S100a9 & 1.925666903 & 1.644888038 \\
\hline Fgf1 & 1.511717006 & 0.859052226 \\
\hline Sapcd2 & 1.462544932 & 0.843682776 \\
\hline Vill & 1.44065248 & 1.168884245 \\
\hline Afmid & 1.428897041 & 1.143546301 \\
\hline Apoc3 & 1.423093388 & 0.852601068 \\
\hline Cox7b2 & 1.418620254 & 1.169692228 \\
\hline Plac9b & 1.412393093 & 0.936219369 \\
\hline $\operatorname{Rec} 114$ & 1.388318026 & 0.853091999 \\
\hline Rgs11 & 1.371627131 & 1.297589728 \\
\hline Rassf6 & 1.35346011 & 0.776802531 \\
\hline Aim1l & 1.300262342 & 0.830519242 \\
\hline Hmga1-rs1 & 1.283781866 & 1.002864541 \\
\hline DII3 & 1.2418496 & 0.915066287 \\
\hline Rpl31-ps10 & 1.239633566 & 0.792724621 \\
\hline Proser3 & 1.21986594 & 0.787241251 \\
\hline Acrbp & 1.215028778 & 1.15765211 \\
\hline Trim30a & 1.210871766 & 1.960530095 \\
\hline Serpina3n & 1.205598425 & 2.354575062 \\
\hline \multicolumn{3}{|c|}{ Downregulated gene name } \\
\hline Snora73a & 0.406488612 & 1.654248839 \\
\hline H3f3aos & 0.541472487 & 0.49289821 \\
\hline $\operatorname{Ttc} 29$ & 0.548897961 & 0.5675665999 \\
\hline Axdnd1 & 0.605272291 & 0.609783214 \\
\hline Shisa6 & 0.621923586 & 0.94496739 \\
\hline Spata1 & 0.634903076 & 0.909699147 \\
\hline Nat8f5 & 0.65271449 & 0.789471856 \\
\hline Poln & 0.653871748 & 0.620157991 \\
\hline Rps15a-ps8 & 0.669896698 & 0.690097602 \\
\hline $\mathrm{Nr} 4 \mathrm{a} 2$ & 0.673787814 & 0.544566896 \\
\hline $\operatorname{Ctgf}$ & 0.681661677 & 0.693422844 \\
\hline Rbm48 & 0.683439751 & 0.665261629 \\
\hline Fam216b & 0.695408168 & 0.685622052 \\
\hline Cfap70 & 0.697868124 & 0.65213551 \\
\hline Dmkn & 0.698743825 & 0.612754578 \\
\hline $\mathrm{E} 2 \mathrm{f3}$ & 0.701420542 & 0.929957578 \\
\hline Fam47e & 0.702211016 & 0.648058675 \\
\hline $0 \mathrm{df3b}$ & 0.711661384 & 0.619959881 \\
\hline Kbtbd4 & 0.721770323 & 0.562695179 \\
\hline Fam117a & 0.73085394 & 1.162965082 \\
\hline
\end{tabular}

The top 20 genes based on relative expression levels of the training + scrambled siRNA group compared with the naive group. The relative gene expression level compared with the naive group for both the training + scrambled siRNA group (scrambled siRNA/naive) and training + BAF53b siRNA group (BAF53b siRNA/naive) are shown. The entire list of differentially expressed genes is in Extended Data Table 1-1.

white opaque Plexiglas and consisted of two open arms $(30 \times 5 \mathrm{~cm})$ and two closed arms $(30 \times 5 \times 15 \mathrm{~cm})$, arranged with identical arms opposite one another. It is raised $50 \mathrm{~cm}$ above the floor. Animals were tested for $5 \mathrm{~min}$. The percentage of time spent in the closed and open arms was scored using EthoVision XT (Noldus).

Histology. Mice were deeply anesthetized with avertin to be perfused and fixed with $4 \%$ paraformaldehyde. The brains were extracted and sliced in coronal sections at $40 \mu \mathrm{m}$ thickness using vibratome (catalog \#VT1000S, Leica). The sections were stained with $1 \%$ Neutral Red. Microscopic examination (ECLIPSE 80i, Nikon) verified the position of injection cannula. The locations of injection cannula tips for mice included in the analyses are presented in Extended Data Figure 2-2. The mice showing cell death were excluded from the data analysis.

Antibodies. The following antibodies were used for Western blot analysis: anti-BAF53b (catalog \# 75-311, NeuroMab), anti-BAF45b (catalog \# ab173160, Abcam), anti-GAPDH (catalog \#ab9485, Abcam,), 
A

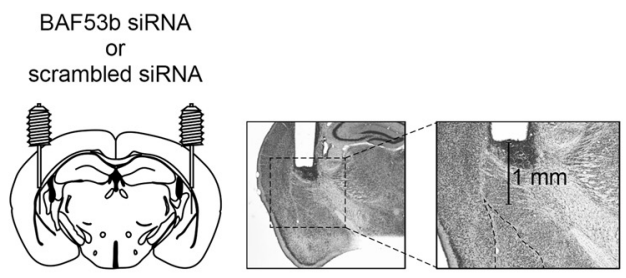

B

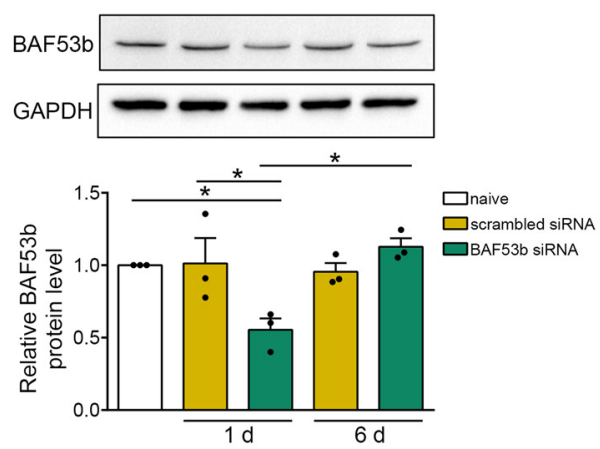

C
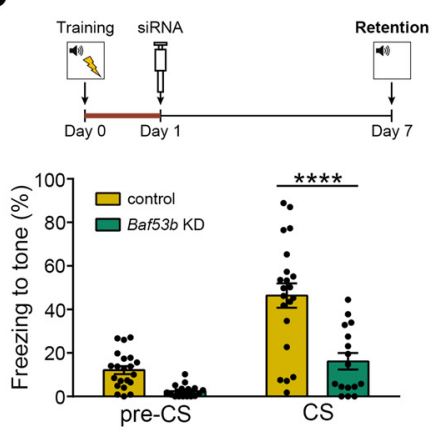

E

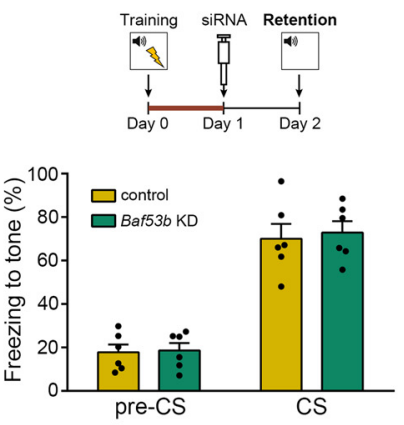

H
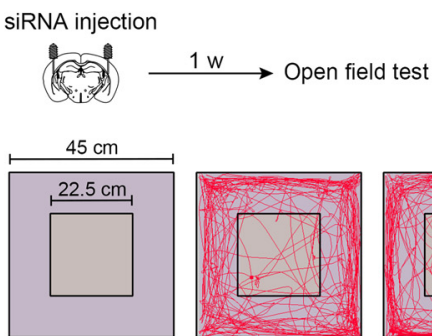

Open field test

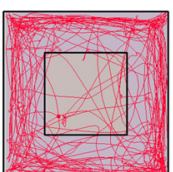

control

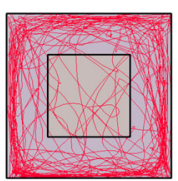

Baf53b KD

D
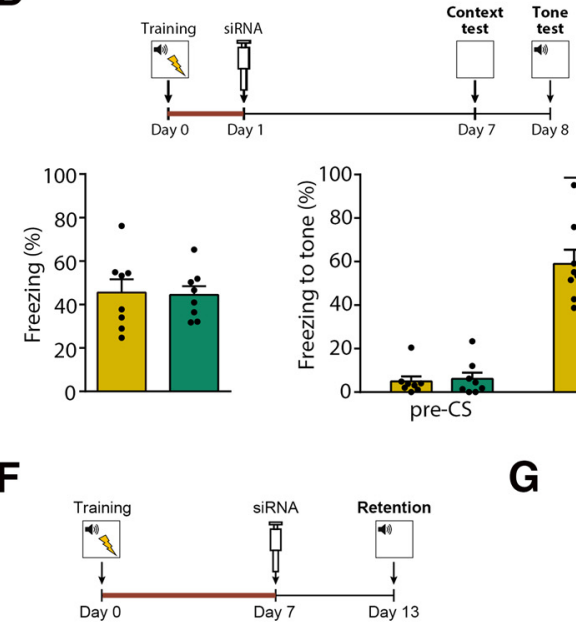

** $\square$ control

F

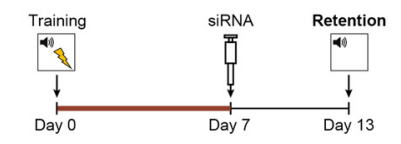

G
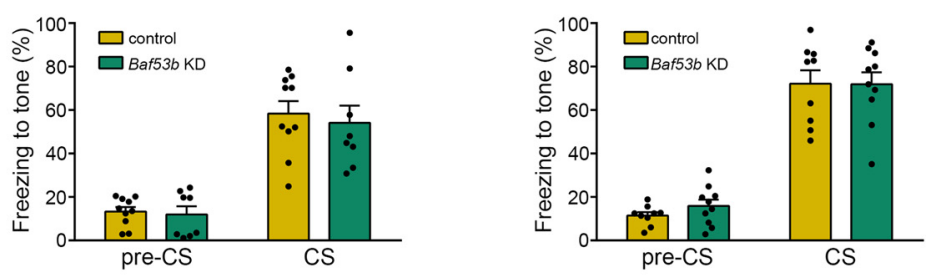

I

$\mathbf{J}$
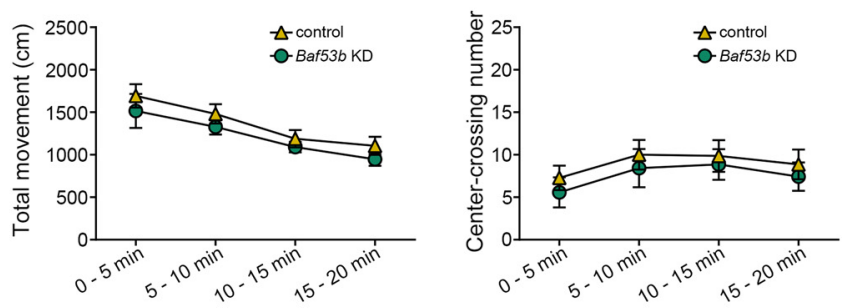

Figure 2. BAF53b siRNA injection at $1 \mathrm{~d}$, but not $7 \mathrm{~d}$, after AFC in the LA specifically impairs remote memory retention. $\boldsymbol{A}$, Representative microscopic image of cannula implantation. Injection cannula was $1 \mathrm{~mm}$ projected below the tip of guide cannula. $\boldsymbol{B}$, Representative Western blots and quantification. The BAF53b protein levels were normalized to the GAPDH protein level ( $n=3$ /group). $\boldsymbol{C}-\boldsymbol{E}$, Histograms showing results from memory retention tests in mice injected with either BAF53b [Baf53b knockdown (KD)] or scrambled (control) siRNA at $1 \mathrm{~d}$ after training. $\boldsymbol{C}$, Retention $6 \mathrm{~d}$ after siRNA injection (control, $n=21 ; B a f 53 b \mathrm{KD}, n=16$ ). $\boldsymbol{D}$, Left, Context test $6 \mathrm{~d}$ after siRNA injection. Right, Tone test $1 \mathrm{~d}$ after context test (control, $n=8 ;$ Baf53b KD, $n=8$ ). $\boldsymbol{E}$, Retention $1 \mathrm{~d}$ after siRNA injection (control, $n=6$; Baf53b KD, $n=6$ ). $\boldsymbol{F}, \mathbf{G}$, Histograms showing results from memory retention tests in mice injected with either BAF53b (Baf53b KD) or scrambled (control) siRNA at $7 \mathrm{~d}$ after training. $\boldsymbol{F}$, Retention $6 \mathrm{~d}$ after siRNA injection (control, $n=10 ;$ Baf53b KD, $n=8$ ). $\boldsymbol{G}$, Retention $1 \mathrm{~d}$ after siRNA injection (control, $n=9 ;$ Baf53b KD, $n=10)$. $\boldsymbol{H}-\boldsymbol{J}$, Open field test with either scrambled (control, $n=7$ ) or BAF53b (Baf53b KD, $n=7$ ) siRNA-injected mice. $\boldsymbol{H}$, Top, Experimental procedure. Bottom, Representative activity pattern tracked by EthoVision XT (Noldus). I, J, Total distance moved ( $\boldsymbol{I}$ ) and center crossings ( $\boldsymbol{J}$ ) during $20 \mathrm{~min}$. Uncropped Western blots are presented in Extended Data Figure 2-1. The locations of injection cannula tips for mice included in the analyses are presented in Extended Data Figure $2-2 A-D . n$ values indicate the number of mice. Data are the mean \pm SEM. ${ }^{*} p<0.05,{ }^{* *} p<$ $0.01,{ }^{* * *} p<0.0001$.

anti-FGF1 (catalog \#ab207321, Abcam), anti-FGFR1 (catalog \#3472, Cell Signaling Technology), anti-phospho-FGFR (catalog \#3476, Cell Signaling Technology), HRP-conjugated anti-mouse IgG light chain (catalog \#AP200P, Millipore), HRP-conjugated anti-mouse IgG (catalog
\#12-349, Millipore), and HRP-conjugated anti-rabbit IgG (catalog \#12348, Millipore) antibodies.

Experimental design and statistical analysis. The experiments used a between-subject design to compare experimental groups and the control 
group. Data are presented as the mean \pm SEM; $n$ values indicate the number of mice. No statistical methods were used to predetermine sample sizes, but our sample sizes are similar to those reported in the previous publication (Yoo et al., 2017). Statistical analyses included two-tailed Student's $t$ tests, one-way ANOVAs followed by Tukey's post hoc test, and repeated-measures two-way ANOVAs followed by Sidak's post hoc test, where appropriate. Statistical analyses were conducted with GraphPad Prism 6 version 6.05. Group differences were considered statistically significant at ${ }^{\star} p<0.05,{ }^{* *} p<0.01,{ }^{* * *} p<0.001$, and ${ }^{* * *} p<$ 0.0001 .

\section{Results}

\section{Elevated level of BAF53b in the LA beyond $24 \mathrm{~h}$ after the auditory fear conditioning}

We first confirmed our previous report of Baf53b mRNA induction in the LA after cued fear learning (Yoo et al., 2017). We conducted either auditory fear conditioning (AFC) or immediate shock as a control on the mice and dissected the LA $6 \mathrm{~h}$ later. The qRT-PCR with reverse transcription result showed that the Baf53b mRNA level increased only in the AFC but not in the footshock group compared with the home cage control (home cage: $1 \pm 0.067, n=9$; immediate shock: $0.87 \pm 0.054, n=5$; AFC: $1.61 \pm 0.29, n=5 ; F_{(2,16)}=6.47, p=0.0087$; one-way ANOVA followed by Tukey's test). We next determined whether the learning-induced Baf53b mRNA returns to the baseline. We conducted AFC on mice and measured Baf53b mRNA levels in the LA 1 or $7 \mathrm{~d}$ after the training. We observed a significant increase at $1 \mathrm{~d}$, but the baseline level at $7 \mathrm{~d}$ (Fig. $1 A ; F_{(2,24)}=$ $10.45, p=0.005$, one-way ANOVA followed by Tukey's test). To further confirm that the Baf53b induction is learning specific, we examined the Baf53b mRNA level in mice trained with either a paired or unpaired conditioning protocol. As before, Baf53b mRNA level was measured in the LA $1 \mathrm{~d}$ after training. We again found a significant increase of Baf53b mRNA level in the paired condition compared with controls, confirming that the late posttraining induction of $B a f 53 b$ is specific to associative learning. (Fig. $1 B ; F_{(2,23)}=4.53, p=0.0219$, one-way ANOVA followed by Tukey's test). Western blot analyses on LA tissues obtained $2 \mathrm{~d}$ after AFC also showed a significantly increased level of BAF53b protein expression in mice from the AFC group compared with the home cage control group (Fig. 1C; two-tailed Student's $t$ test, $\left.t_{(10)}=4.029, p=0.0024\right)$. Previous studies reported similarly late induction for several other genes involved in the memory persistence such as BDNF and immediate early genes in different learning tasks (Katche et al., 2013; Nakayama et al., 2016). However, as far as we know, there is no report of such a late-phase increase of expression for epigenetic regulators. Therefore, we investigated whether the late induced BAF53b is involved in persistent retention of fear memory.

\section{Baf53b knockdown in the LA $1 \mathrm{~d}$ after AFC specifically impairs remote memory retention}

We used an RNAi method that allowed us to transiently knock down Baf53b in the LA after learning. First, to confirm Baf53b knockdown efficiency by siRNA, we performed Western blotting on LA tissues obtained 1 or $6 \mathrm{~d}$ after cannula injection of BAF53b siRNA (see Materials and Methods) into the bilateral LA (Fig. 2A). Scrambled siRNA-injected or naive mice were used as controls. Western blot analyses showed that the BAF53b protein level decreased by $\sim 50 \%$ at $1 \mathrm{~d}$ but returned to baseline level at $6 \mathrm{~d}$ after siRNA injection (Fig. $2 B ; F_{(4,10)}=5.49, p=0.013$, oneway ANOVA followed by Tukey's test), indicating Baf53b knockdown by siRNA is efficient and temporally reversible.
For behavioral tests, mice implanted with a cannula were trained with AFC, and the next day administered with either BAF53b or control scrambled siRNA into the bilateral LA and then were tested for memory retention $6 \mathrm{~d}$ later. Mice in the $B a f 53 b$ knock-down group showed significantly reduced freezing compared with the control group (Fig. $2 C ; F_{(1,35)}=10.18$, $p=0.0030$, two-way repeated-measures ANOVA followed by Sidak's test). This reduced freezing was not because of the loss of learning ability. The Baf53b knock-down mice could relearn the tone-shock association by retraining. When the same mice were retrained $1 \mathrm{~d}$ after day 7 retention and tested $1 \mathrm{~d}$ later, they showed a normal conditioned freezing response (pre-CS, $14.45 \pm 2.50 \%$; CS, $47.95 \pm 4.38 \% ; n=16$; two-tailed Student's $t$ test, $\left.t_{(30)}=6.645, p<0.0001\right)$. To test the specificity of the $B a f 53 b$ knock-down effect, we performed a contextual fear memory test. BAF53b siRNA was injected into the bilateral LA $1 \mathrm{~d}$ after auditory fear conditioning, and mice were tested for contextual fear memory $6 \mathrm{~d}$ later. Next day, mice were tested for auditory cued fear memory. Mice injected with scrambled siRNA were included as a control for comparison. We observed no significant difference in context-dependent freezing between groups, whereas auditory cued freezing was significantly reduced in the BAF53b siRNA-injected mice compared with control mice as we found before. This result indicates that the effect of Baf53b knockdown in the LA is specific to auditory cued fear memory (Fig. 2D; context test: two-tailed Student's $t$ test, $t_{(14)}=0.15$, $p=0.88$; tone test: $F_{(1,14)}=12.06, p=0.0037$, two-way repeatedmeasures ANOVA followed by Sidak's test). The Baf53b knockdown effect was specific to memory retention at the remote time. Mice showed normal memory retention if tested $1 \mathrm{~d}$ after siRNA injection (Fig. $2 E ; F_{(1,10)}=0.11, p=0.74$, two-way repeatedmeasures ANOVA). This result suggests that the late induction of BAF53b is not involved in recent memory expression.

To further test the specificity of the Baf53b knock-down effect, this time we injected BAF53b siRNA at $7 \mathrm{~d}$ after training. We found no significant differences in conditioned freezing between groups during both 6 and $1 \mathrm{~d}$ postinjection memory tests (Fig. $2 F, G$ : $F_{(1,16)}=0.20, p=0.66$, two-way repeated-measures ANOVA; Fig. $2 G$ : $F_{(1,16)}=0.27, p=0.60$, two-way repeatedmeasures ANOVA). The open field test confirmed no significant difference in locomotor activity and anxiety level between BAF53b and control scrambled siRNA-injected mice (Fig. $2 \mathrm{H}-\mathrm{J}$; Fig. 2I: $F_{(3,36)}=0.054, p=0.98$; Fig. $2 J: F_{(3,36)}=0.036, p=0.99$; two-way repeated-measures ANOVA). These control data together support that the reduced freezing during the remote memory test was not because of impaired auditory function or an abnormal increase of locomotor activity and basal anxiety. Nevertheless, our data do not completely rule out the possibility that the intra-LA knockdown of Baf53b might influence certain aspects of auditory function.

\section{$B a f 45 b$ knockdown has no effect on the remote memory retention}

The mammalian BAF complexes are made up of 15 subunits (Ronan et al., 2013). Among them, a double-PHD (plant homeodomain) subunit BAF45b is similar to BAF53b in that it is a stoichiometric component of nBAF complex specifically expressed in postmitotic neurons (Lessard et al., 2007) and is essential for activity-dependent dendritic growth (Wu et al., 2007). Thus, we asked whether BAF45b also affects remote memory formation. By conducting Western blot analyses, we first found that, unlike BAF53b, does not show late induction when examined at $2 \mathrm{~d}$ after training (Fig. 3A; two-tailed Student's $t$ test, $t_{(10)}=0.34$, 
A
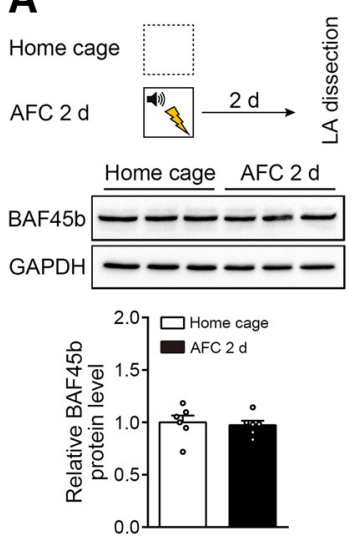

B

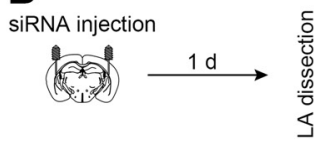

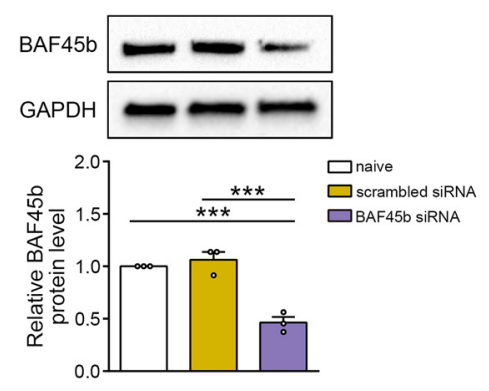

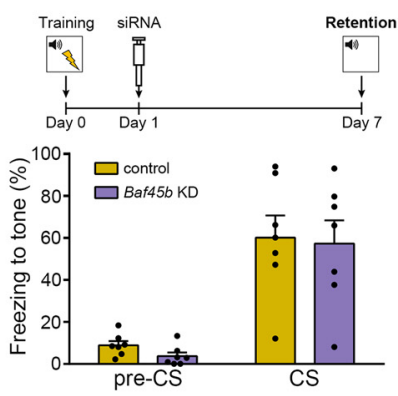

Figure 3. No effect of Baf45b knockdown (KD) on remote memory retention. $A, B$, Representative Western blots and quantification results. The BAF45b protein levels were normalized to the GAPDH protein level. $\boldsymbol{A}, \mathrm{BAF} 45 \mathrm{~b}$ protein level $2 \mathrm{~d}$ after AFC ( $n=6 /$ group). $\boldsymbol{B}, \mathrm{BAF} 45 \mathrm{~b}$ protein level $1 \mathrm{~d}$ after siRNA injection ( $n=3 /$ group). $\boldsymbol{C}$, Top, Behavioral procedure. Bottom, Toneinduced freezing during retention test. Mice were injected with either scrambled siRNA (control, $n=7$ ) or BAF45b siRNA (Baf45b KD, $n=7$ ) $1 \mathrm{~d}$ after training and tested for memory retention $6 \mathrm{~d}$ later. Uncropped Western blots are presented in Extended Data Figure 3-1. The locations of injection cannula tips for mice included in the analyses are presented in Extended Data Figure $2-2 E . n$ values indicate the number of mice. Data are the mean \pm SEM. ${ }^{* *} p<0.001$.

$p=0.74)$. To compare with BAF53b, we again used the RNAi method for the Baf53b test. Western blot analyses confirmed the efficient knockdown of Baf $45 b$ by the target siRNA but not by scrambled siRNA compared with naive control. BAF45b protein level decreased by $\sim 50 \%$ when measured at $1 \mathrm{~d}$ after siRNA injection (Fig. $3 B ; F_{(2,6)}=38.16, p=0.0004$, one-way ANOVA followed by Tukey's test). For the behavioral test, as before, we injected either BAF45b or scrambled siRNA into the bilateral LA of two separate groups of mice $1 \mathrm{~d}$ after AFC and tested the mice $6 \mathrm{~d}$ later. We found no significant group difference in freezing during the test, highlighting the specific role of BAF53b (Fig. 3C; $F_{(1,12)}=0.024, p=0.88$, two-way repeated-measures ANOVA).

FGF1 peptide injection rescues remote memory impairment by $B a f 53 b$ knockdown

Given that BAF53b is a nucleosome remodeling factor, we next sought genes affected by Baf53b knockdown among those upregulated or downregulated at the remote time after AFC by performing RNA-seq experiment. The LA tissues obtained from three groups of mice (Fig. $4 A$; naive, scrambled siRNA, and BAF53b siRNA) were used. Of the differentially expressed genes in the training + scrambled siRNA group compared with the naive group (Table 1), we focused on FGF1 as a candidate for downstream effector based on previous reports showing its involvement in neurite outgrowth (Raju et al., 2014) and memory formation (Uchida et al., 2017). Fgf1 gene expression was induced by the training, whereas such upregulation was abolished by Baf53b knockdown (Fig. $4 A$ ), which was further confirmed by qRT-PCR analysis (Fig. $4 B ; F_{(2,12)}=7.019, p=0.0096$, one-way ANOVA followed by Tukey's test). To further confirm that the Fgf1 mRNA increase is learning specific, we examined the $F g f 1$ mRNA level in mice trained with either paired or unpaired conditioning protocol. As before, the Fgf1 mRNA level was measured in the LA $7 \mathrm{~d}$ after training. We again found a significant increase in Fgfl mRNA level in the paired condition compared with controls, confirming that the late post-training induction of $\mathrm{Fg} 1 \mathrm{i}$ is specific to associative learning. (Fig. 4C; $F_{(2,21)}=7.87, p=0.0028$, one-way ANOVA followed by Tukey's test). Based on the RNA-seq result, we next performed behavioral rescue experiments to test whether applying FGF1 peptide can prevent memory loss at the remote time in mice with Baf53b knockdown. We used the same behavioral procedures as before except that FGF1 peptide was locally injected into the bilateral LA ( $5 \mathrm{ng} /$ each side) $1 \mathrm{~d}$ after siRNA injection. As before, the Baf53b knockdown impaired remote memory retention. However, the Baf53b knock-down mice that received FGF1 peptide but not vehicle showed a similar level of freezing with control mice, suggesting the rescue of memory loss by FGF1 (Fig. $4 D ; F_{(2,25)}=8.78, p=0.0013$, two-way repeated-measures ANOVA followed by Sidak's test). To examine the elevation of FGF1, we measured the amount of FGF1 and also the phosphorylation of FGFR1, a predominant FGF1 receptor in the amygdala (Allen Brain Atlas; Belluardo et al., 1997; Lein et al., 2007), as an index of the activation of FGF1 signaling (Nies et al., 2016) using Western blots. Based on a prior in vitro report on temporal dynamics of FGFR1 phosphorylation (Huang et al., 2017), the bilateral LA tissues were obtained 15 min after FGF1 peptide or control vehicle injection. We found that there was a significant increase (23\% increase) in the level of FGF1 (Fig. 4E; two-tailed Student's $t$ test, $\left.t_{(10)}=2.82, p=0.018\right)$. Using brain lysates from the same mice, we measured the level of the pFGFR1. We found a significant increase (120\% increase) in mice with FGF1 peptide injection compared with control mice (Fig. 4F; two-tailed Student's $t$ test, $\left.t_{(10)}=5.22, p=0.0004\right)$. These results demonstrate the elevation of the FGF1 level by peptide injection in the in vivo brain. In addition, we performed an open field test (Fig. $4 G-I$ ) and elevated plus maze (Fig. $4 J, K$ ) to determine whether the FGF1 peptide infusion in the LA affects basal anxiety or locomotor behavior. We found no significant difference between groups in both tests. (Fig. $4 H: F_{(3,24)}=0.52, p=0.68$; Fig. 4I: $F_{(3,24)}=0.24, p=0.86$, two-way repeated-measures ANOVA; Fig. $4 K: F_{(1,14)}=0.036, p=0.85$, two-way ANOVA).

To determine the specificity of the effect of FGF1, we repeated the same behavioral test, but this time with FGF2 peptide, another member of the same FGF subfamily that has the highest amino acid sequence homology (54\%) with FGF1 among other FGFs. While FGF1 binds to all known FGFR subtypes on the cell surface (i.e., FGFR1b, FGFR1c, FGFR2b, FGFR2c, FGFR3b, FGFR3c, and FGFR4), FGF2 binds with most of the FGFR subtypes except for FGFR2b and FGFR3b (Ornitz et al., 1996) and is shown to be involved in neurogenesis, and fear memory acquisition and extinction in developing and adult rats (Graham and 
A

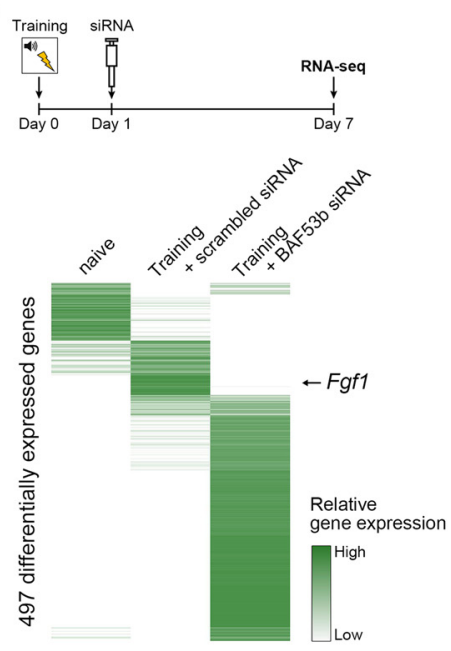

D
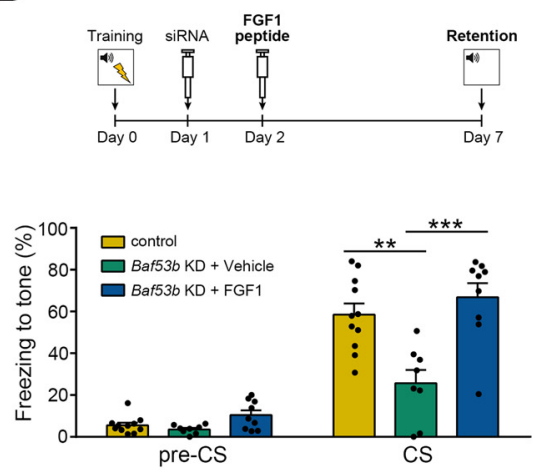

G

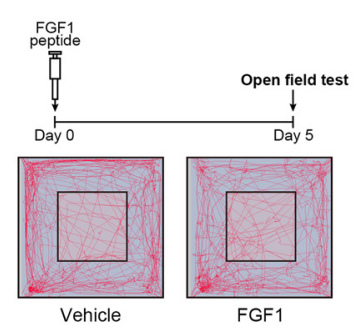

J

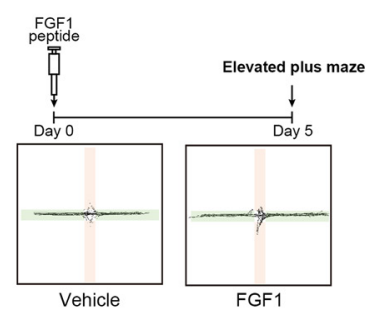

B

C
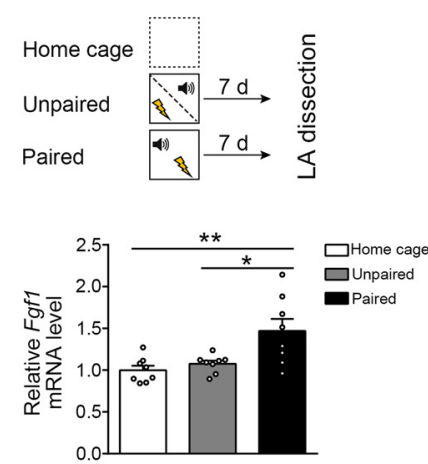

E
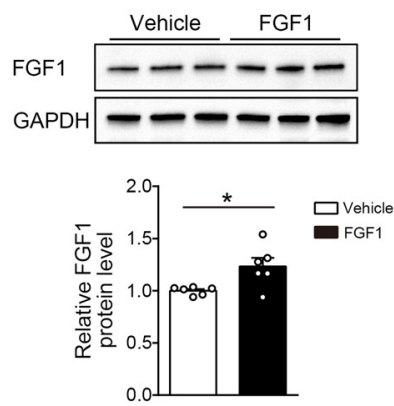

H

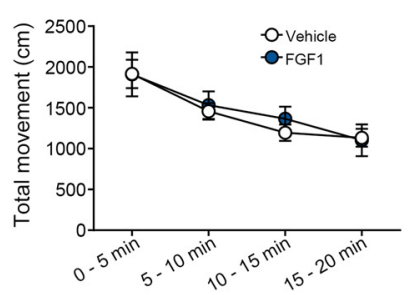

$\mathbf{K}$

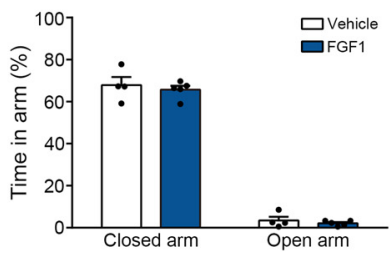

$\mathbf{F}$
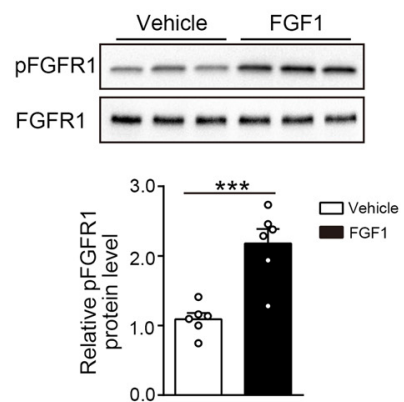

I

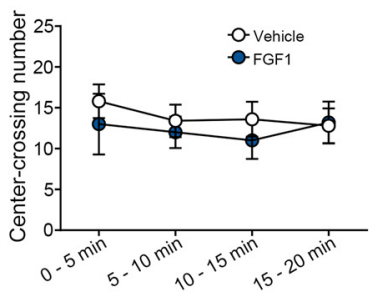

Figure 4. FGF1 peptide injection prevents remote memory loss by Baf53b knockdown. A, Top, Experimental procedure. Bottom, A heatmap showing relative expression levels of differentially expressed genes in naive mice and mice receiving auditory fear conditioning with either BAF53b siRNA or scrambled siRNA injection $1 \mathrm{~d}$ after the training $(n=2 / g r o u p ; ~ L A$ tissues from 4 mice were collected for each $n$ value). $\boldsymbol{B}$, Top, Experimental procedures. Bottom, qRT-PCR results showing relative Fgf1 mRNA levels compared with naive control measured $6 \mathrm{~d}$ after siRNA injection in mice trained with AFC (Training). The Fgf1 mRNA levels were normalized to the Gapdh mRNA level ( $n=5 /$ group) $C$, Top, Experimental procedures. Bottom, qRT-PCR results showing relative Fgf1 mRNA levels compared with home cage control measured $7 \mathrm{~d}$ after unpaired or paired conditioning. The Fgf1 mRNA levels were normalized to the Gapdh mRNA level ( $n=8 / \mathrm{group})$. $\boldsymbol{D}$, Top, Experimental procedure. Bottom, Tone-induced freezing during retention test. Control, Scrambled siRNA injection $(n=11)$; Baf53b KD + Vehicle, BAF53b siRNA and vehicle $(0.5 \mu l$ of $0.1 \%$ BSA per each side) injection $(n=8)$. Baf53b KD + FGF1, BAF53b siRNA, and FGF1 peptide $(0.5 \mu \mathrm{l}$ of $10 \mathrm{ng} / \mu$ l peptide solution per each side; $n=9)$. $\boldsymbol{E}, \boldsymbol{F}$, Representative Western blots and quantification results for FGF1 and FGFR1( $n=6$ /group). $\boldsymbol{E}$, FGF1 protein level normalized to the GAPDH protein level. $\boldsymbol{F}$, pFGFR1 protein level normalized to the FGFR1 protein level. $\mathbf{G}-\boldsymbol{I}$, Open field test with either vehicle-injected (Vehicle, $n=5$ ) or FGF1 peptide-injected (FGF1, $n=5$ ) mice. G, Top, Experimental procedure. Bottom, Representative activity pattern tracked by EthoVision XT (Noldus). $\boldsymbol{H}, \boldsymbol{I}$, Total distance moved $(\boldsymbol{H})$ and center crossings $(\boldsymbol{I})$ during $20 \mathrm{~min}$. $\boldsymbol{J}, \boldsymbol{K}$, Elevated plus maze with either vehicle-injected (Vehicle, $n=5)$ or FGF1 peptide-injected $(\mathrm{FGF1}, n=5)$ mice. $\boldsymbol{J}$, Top, Experimental procedure. Bottom, Representative activity pattern tracked by EthoVision XT (Noldus). $\boldsymbol{K}$, Percentage of time in closed or open arm during 5 min. Uncropped Western blots are presented in Extended Data Figure 4-1. The locations of injection cannula tips for mice included in the analyses are presented in Extended Data Figure 2-2F. $n$ values indicate the number of mice, except for Figure $4 A$. Data are the mean \pm SEM. ${ }^{*} p<0.05,{ }^{* *} p<0.01,{ }^{* * *} p<0.001$. 
Richardson, 2011). According to our RNA-seq result, unlike $F g f 1$, there was no significant change of $\mathrm{Fg} 2$ expression in the LA in training and Baf53b knock-down conditions, which was also confirmed by qRT-PCR (Fig. $5 A$; $F_{(2,12)}=0.81, \quad p=0.47$, one-way ANOVA). In the behavior experiment, FGF2 peptide was injected into the bilateral LA as the FGF1 experiment, but this time we did not observe any significant effect, thus supporting the specific effect of FGF1 (Fig. $5 B ; F_{(2,18)}$ $=5.94, p=0.011$, two-way repeatedmeasures ANOVA followed by Sidak's test).

\section{Discussion}

Altogether, our results reveal the crucial role of BAF53b and FGF1 in the regulation of fear memory persistence. No memory loss at the recent time in Baf53b knock-down mice suggests that elevated BAF53b expression is unlikely to be the time-dependent temporary memory storage mechanism. Moreover, no effect of Baf53b knockdown $7 \mathrm{~d}$ after learning supports the temporary role of BAF53b for memory retention. These findings are consistent with the idea that the late increased BAF53b is a time-dependent consolidation mechanism enabling persistent retention of memory.

One of the novel findings in this study is the late post-training increase of BAF53b expression in the LA after auditory fear conditioning. Previous studies reported similarly delayed induction for several other genes such as BDNF and immediate early genes in different learning tasks (Katche et al., 2013; Nakayama et al., 2016). However, as far as we know, our study is the first report of such a late-phase increase in gene expression for epigenetic regulators. Accumulating evidence strongly supports that the late wave of gene expression modifications is required for the persistence of long-term memory (Katche et al., 2013). So, it is becoming clear that cellular consolidation requires not only early but also late post-training events of gene expression changes. Our findings in this study are consistent with this hypothesis. It is unclear how BAF53b is induced even long after learning. Epigenetic regulation such as histone modifications is one possible mechanism. Alternatively, as previously proposed, an offline internal replay of neural activities present during learning may underlie the late BAF53b induction (Bekinschtein et al., 2007).

Numerous studies have shown that structural changes of synapses are involved in long-term memory storage in various forms of memory (Bailey et al., 2004; Lamprecht and LeDoux, 2004; Segal, 2005). Previous works including ours suggest that BAF53b may contribute to the consolidation of memory by regulating spine structural plasticity via actin signaling pathway. The in vitro study using cultured hippocampal neurons shows that BAF53b is critical for activity-dependent dendritic outgrowth (Wu et al., 2007). Alterations of dendritic spine morphology and activity-dependent phosphorylation of Cofilin have been reported in the hippocampal neurons of Baf53b heterozygous knock-out mice and transgenic mice that express a mutant BAF53b with a deletion of the hydrophobic domain (VogelCiernia et al., 2013). In these mice, several key postsynaptic density genes involved in spine plasticity show altered expression. In
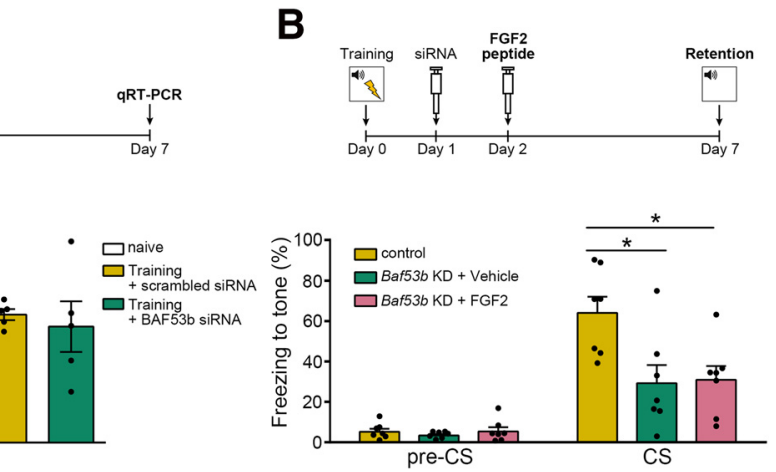

cit by Baf53b knockdown. $\boldsymbol{A}$, Top, Experimental procedure. Bottom, qRT-PCR results showing relative Fgf2 mRNA levels compared with naive control measured $6 \mathrm{~d}$ after siRNA injection in mice trained with AFC (Training). The Fgf2 mRNA levels were normalized to the Gapdh mRNA level ( $n=5 / g r o u p)$. $\boldsymbol{B}$, Top, Experimental procedure. Bottom, Tone-induced freezing during retention test. Control $(n=7)$, Scrambled siRNA injection; Baf53b KD + Vehicle $(n=7)$, BAF53b siRNA and vehicle $(0.5 \mu \mathrm{l}$ of $0.1 \%$ BSA per each side) injection; and Baf53b KD + FGF2 $(n=7)$, FiRNA and FGF2 peptide $(0.5 \mu \mathrm{l}$ of $10 \mathrm{ng} / \mu \mathrm{l}$ peptide solution per each side) injection. The locations of injection cannula tips for mice included in the analyses are presented in Extended Data Figure 2-2G. $n$ values indicate the number of mice. Data

our previous work, BAF53b overexpression promoted thin spine outgrowth in the LA neurons of wild-type mice following auditory fear conditioning (Yoo et al., 2017). Based on these findings, we hypothesize that the late post-training increase of BAF53b expression even long after learning may be required for long-term stabilization of learning-induced synapse structural modifications that support remote memory storage. Investigation of spine density or morphological changes combined with the approaches we used in this study will help address this idea in the future study. Although we focused on the LA in this study, previous studies suggest that BAF53b plays a crucial role in different brain regions for different forms of memory (VogelCiernia et al., 2013; White et al., 2016). It is unclear at this time whether the late induction of BAF53b is a common mechanism for the persistence of memory. Although no significant induction of BAF53b has been reported early phase after learning in the hippocampus (Vogel-Ciernia et al., 2013), it is still possible that BAF53b is induced at the late phase.

The results from RNA-seq analyses and behavioral rescue experiments identified FGF1 as a candidate downstream effector mediating BAF53b function for the persistence of fear memory. A prior study indicates that BAF53b is not required for neuronal BAF (nBAF) complex assembly or interaction with CREST, a $\mathrm{Ca}^{2+}$-responsive transcriptional coactivator, yet is required for their recruitment to the promoters of specific target genes $(\mathrm{Wu}$ et al., 2007). Given that BAF53b is a stoichiometric component of the combinatorial assembly of BAF complex (Olave et al., 2002; Wu et al., 2007), a possible scenario is that the induction of BAF53b may promote the assembly of nBAF complex containing the BAF53b and thereby the recruitment of nBAF complex to the promoter of Fgfl gene in the LA neurons. Consistent with our findings in this study, a recent study in the mouse hippocampus shows that FGF1 enhances the maintenance of synaptic plasticity and improves associative contextual fear memory. Interestingly, temporal regulation of $F g f l b$ gene expression is shown to be correlated with the strength of associative memory such that a weak training protocol leads to transient increase of FGF1, whereas strong training leads to the sustained increase of FGF1 (Uchida et al., 2017).

According to our RNA-seq data, in addition to FGF1, there are several other genes whose upregulation or downregulation 
$7 \mathrm{~d}$ after auditory fear conditioning were altered by Baf53b knockdown. There are two genes that are upregulated above Fgf1: Scgb3a1 and S100a9 (Table 1). Their expression increased $>1.9$ times after training. However, Baf53b knockdown only slightly diminished the increase to 1.4-1.6 times. So, we speculated that these genes may not be critically involved in the mechanism of BAF53b action on memory. Among the genes downregulated after training, Shisa6 is a promising candidate downstream effector of BAF53b (Table 1). Shisa6 has been identified as a stable and directly interacting AMPA receptor (AMPAR) auxiliary subunit. Shisa6 is thought to be involved in activity-dependent regulation of AMPAR concentration at synapses by limiting AMPAR diffusion and stabilizing AMPAR at synaptic sites (Klaassen et al., 2016).

In this study, our results reveal the crucial role of BAF53b and FGF1 in the regulation of fear memory persistence. This study gives insights into how fear memory persists through consolidation processes involving nucleosome remodeling in the amygdala, which is highly relevant to fear-related mental disorders.

\section{References}

Albo Z, Gräff J (2018) The mysteries of remote memory. Philos Trans R Soc Lond B Biol Sci 373:20170029.

Bailey CH, Kandel ER, Si K (2004) The persistence of long-term memory: a molecular approach to self-sustaining changes in learning-induced synaptic growth. Neuron 44:49-57.

Bekinschtein P, Cammarota M, Igaz LM, Bevilaqua LRM, Izquierdo I, Medina JH (2007) Persistence of long-term memory storage requires a late protein synthesis- and BDNF-dependent phase in the hippocampus. Neuron 53:261-277.

Belluardo N, Wu G, Mudo G, Hansson AC, Pettersson R, Fuxe K (1997) Comparative localization of fibroblast growth factor receptor-1, -2 , and -3 mRNAs in the rat brain: in situ hybridization analysis. J Comp Neurol 379:226-246.

Campbell RR, Wood MA (2019) How the epigenome integrates information and reshapes the synapse. Nat Rev Neurosci 20:133-147.

Day JJ, Sweatt JD (2011) Epigenetic mechanisms in cognition. Neuron 70:813-829.

DiCarlo LM, Vied C, Nowakowski RS (2017) The stability of the transcriptome during the estrous cycle in four regions of the mouse brain. J Comp Neurol 525:3360-3387.

Dudai Y, Karni A, Born J (2015) The consolidation and transformation of memory. Neuron 88:20-32.

Graham BM, Richardson R (2009) Acute systemic fibroblast growth factor-2 enhances long-term memory in developing rats. Neurobiol Learn Mem 91:424-430.

Graham BM, Richardson R (2011) Memory of fearful events: the role of fibroblast growth factor-2 in fear acquisition and extinction. Neuroscience 189:156-169.

Huang Z, Tan Y, Gu J, Liu Y, Song L, Niu J, Zhao L, Srinivasan L, Lin Q, Deng J, Li Y, Conklin DJ, Neubert TA, Cai L, Li X, Mohammadi M (2017) Uncoupling the mitogenic and metabolic functions of FGF1 by tuning FGF1-FGF receptor dimer stability. Cell Rep 20:1717-1728.

Katche C, Cammarota M, Medina JH (2013) Molecular signatures and mechanisms of long-lasting memory consolidation and storage. Neurobiol Learn Mem 106:40-47.

Klaassen RV, Stroeder J, Coussen F, Hafner A-S, Petersen JD, Renancio C, Schmitz LJM, Normand E, Lodder JC, Rotaru DC, Rao-Ruiz P, Spijker S, Mansvelder HD, Choquet D, Smit AB (2016) Shisa6 traps AMPA receptors at postsynaptic sites and prevents their desensitization during synaptic activity. Nat Commun 7:10682.

Lamprecht R, LeDoux J (2004) Structural plasticity and memory. Nat Rev Neurosci 5:45-54.
Lein ES, Hawrylycz MJ, Ao N, Ayres M, Bensinger A, Bernard A, Boe AF, Boguski MS, Brockway KS, Byrnes EJ, Chen L, Chen L, Chen T-M, Chin MC, Chong J, Crook BE, Czaplinska A, Dang CN, Datta S, Dee NR, et al (2007) Genome-wide atlas of gene expression in the adult mouse brain. Nature 445:168-176.

Lessard J, Wu JI, Ranish JA, Wan M, Winslow MM, Staahl BT, Wu H, Aebersold R, Graef IA, Crabtree GR (2007) An essential switch in subunit composition of a chromatin remodeling complex during neural development. Neuron 55:201-215.

McGaugh JL (2000) Memory-a century of consolidation. Science 287:248251.

Merlo E, Bekinschtein P, Jonkman S, Medina JH (2015) Molecular mechanisms of memory consolidation, reconsolidation, and persistence. Neural Plast 2015:687175.

Nakayama D, Hashikawa-Yamasaki Y, Ikegaya Y, Matsuki N, Nomura H (2016) Late Arc/Arg3.1 expression in the basolateral amygdala is essential for persistence of newly-acquired and reactivated contextual fear memories. Sci Rep 6:21007.

Nies VJM, Sancar G, Liu W, Zutphen T, Struik D, Yu RT, Atkins AR, Evans RM, Jonker JW, Downes MR (2016) Fibroblast growth factor signaling in metabolic regulation. Front Endocrinol (Lausanne) 6:193.

Olave I, Wang W, Xue Y, Kuo A, Crabtree GR (2002) Identification of a polymorphic, neuron-specific chromatin remodeling complex. Genes Dev 16:2509-2517.

Ornitz DM, Xu J, Colvin JS, McEwen DG, MacArthur CA, Coulier F, Gao G, Goldfarb M (1996) Receptor specificity of the fibroblast growth factor family. J Biol Chem 271:15292-15297.

Raju R, Palapetta SM, Sandhya VK, Sahu A, Alipoor A, Balakrishnan L, Advani J, George B, Kini KR, Geetha NP, Prakash HS, Prasad TSK, Chang Y-J, Chen L, Pandey A, Gowda H (2014) A network map of FGF1/FGFR signaling system. J Signal Transduct 2014:962962.

Ronan JL, Wu W, Crabtree GR (2013) From neural development to cognition: unexpected roles for chromatin. Nat Rev Genet 14:347-359.

Schoberleitner I, Mutti A, Sah A, Wille A, Gimeno-Valiente F, Piatti P, Kharitonova M, Torres L, López-Rodas G, Liu JJ, Singewald N, Schwarzer C, Lusser A (2019) Role for chromatin remodeling factor Chd1 in learning and memory. Front Mol Neurosci 12:3.

Segal M (2005) Dendritic spines and long-term plasticity. Nat Rev Neurosci 6:277-284.

Uchida S, Teubner BJW, Hevi C, Hara K, Kobayashi A, Dave RM, Shintaku T, Jaikhan P, Yamagata H, Suzuki T, Watanabe Y, Zakharenko SS, Shumyatsky GP (2017) CRTC1 nuclear translocation following learning modulates memory strength via exchange of chromatin remodeling complexes on the Fgf1 gene. Cell Rep 18:352-366.

Vogel-Ciernia A, Matheos DP, Barrett RM, Kramár EA, Azzawi S, Chen Y, Magnan CN, Zeller M, Sylvain A, Haettig J, Jia Y, Tran A, Dang R, Post RJ, Chabrier M, Babayan AH, Wu JI, Crabtree GR, Baldi P, Baram TZ, et al. (2013) The neuron-specific chromatin regulatory subunit BAF53b is necessary for synaptic plasticity and memory. Nat Neurosci 16:552-561.

White AO, Kramár EA, López AJ, Kwapis JL, Doan J, Saldana D, Davatolhagh MF, Alaghband Y, Blurton-Jones M, Matheos DP, Wood MA (2016) BDNF rescues BAF53b-dependent synaptic plasticity and cocaine-associated memory in the nucleus accumbens. Nat Commun 7:11725.

Wu JI, Lessard J, Olave IA, Qiu Z, Ghosh A, Graef IA, Crabtree GR (2007) Regulation of dendritic development by neuron-specific chromatin remodeling complexes. Neuron 56:94-108.

Yang Y, Yamada T, Hill KK, Hemberg M, Reddy NC, Cho HY, Guthrie AN, Oldenborg A, Heiney SA, Ohmae S, Medina JF, Holy TE, Bonni A (2016) Chromatin remodeling inactivates activity genes and regulates neural coding. Science 353:300-305.

Yoo M, Choi K-Y, Kim J, Kim M, Shim J, Choi J-H, Cho H-Y, Oh J-P, Kim H-S, Kaang B-K, Han J-H (2017) BAF53b, a neuron-specific nucleosome remodeling factor, is induced after learning and facilitates long-term memory consolidation. J Neurosci 37:3686-3697. 